\title{
ARTICLE OPEN Effectiveness of case finding strategies for COPD in primary care: a systematic review and meta-analysis
}

\author{
Shamil MM Haroon ${ }^{1}$, Rachel E Jordan ${ }^{1}$, Joanne O'Beirne-Elliman ${ }^{1}$ and Peymane Adab ${ }^{1}$
}

\begin{abstract}
BACKGROUND: Chronic obstructive pulmonary disease (COPD) is widely underdiagnosed, but the most effective approach for identifying these patients is unknown.

AIMS: The aim of this study was to summarise and compare the effectiveness of different case finding approaches for undiagnosed COPD in primary care.

METHODS: A systematic review of primary studies of any design evaluating case finding strategies for COPD in primary care among individuals aged $\geqslant 35$ years with no prior diagnosis was conducted. Medline, Embase and other bibliographic databases were searched from 1997 to 2013, and methodological quality was assessed using standard tools. Results were described and metaanalysis of the uptake and yield from different approaches was performed where there was sufficient homogeneity.

RESULTS: Three randomised controlled trials (RCTs), 1 controlled trial and 35 uncontrolled studies were identified that assessed the identification of new cases of COPD through systematic case finding. A range of approaches were used including pre-screening with questionnaires $(n=13)$ or handheld flow meters $(n=5)$ or direct invitation to diagnostic spirometry $(n=30)$. Overall, any approach identified more undiagnosed COPD compared with usual care. Targeting those at higher risk (e.g., smokers) and pre-screening (e.g., using questionnaires) is likely to increase the yield. However, studies were heterogeneous and were limited by a lack of comparison groups, inadequate reporting and diversity in the definition of COPD, which limited our ability to draw firm conclusions.

CONCLUSIONS: There is extensive heterogeneity among studies evaluating case finding strategies for COPD, with few RCTs. Well-conducted RCTs comparing case finding approaches are needed to identify the most effective target population, recruitment strategy and screening tests, using a clinical definition of COPD, and addressing the limitations highlighted in this review. There is also a need to evaluate the impact of case finding on clinical care and patient outcomes.
\end{abstract}

npj Primary Care Respiratory Medicine (2015) 25, 15056; doi:10.1038/npjpcrm.2015.56; published online 27 August 2015

\section{INTRODUCTION}

Chronic obstructive pulmonary disease (COPD) is the third leading cause of mortality, ${ }^{1}$ an important cause of disability ${ }^{2}$ and a source of significant healthcare expenditure. ${ }^{3}$ However, up to $70-90 \%$ of the disease burden remains undiagnosed. ${ }^{4}$ People with undiagnosed COPD often under-recognise the significance of their symptoms, ${ }^{5}$ and there is poor awareness of the condition among the general public. ${ }^{6,7}$ Clinicians in primary care also frequently miss opportunities to diagnose COPD. ${ }^{8}$ Furthermore, a higher prevalence of undiagnosed COPD has been associated with higher rates of COPD-related hospitalisations, ${ }^{9}$ with diagnosis often being made during an acute hospital admission following an exacerbation. ${ }^{10}$ There is now a policy drive to identify undiagnosed COPD earlier in the course of the disease. ${ }^{11}$ However, the optimal strategy for achieving this remains unknown. ${ }^{12}$

A systematic review published in 2008 evaluated the effectiveness of population-based screening for COPD using spirometry but concluded that this would identify many asymptomatic individuals with mild-to-moderate airflow obstruction, for whom there are limited therapeutic options. ${ }^{13}$ However, since then there have been a large number of studies of potentially more efficient approaches, including the use of screening questionnaires ${ }^{14}$ and handheld flow meters (e.g., Piko-6, Longmont, CO, USA or COPD-6, Buckingham, UK $)^{15}$ and more studies seeking individuals with clinical symptoms.
We report a systematic review to identify and compare the effectiveness (yield) of alternative case finding approaches for COPD in primary care.

\section{MATERIALS AND METHODS}

Protocol and registration

The protocol for this review was previously published ${ }^{16}$ and registered on the PROSPERO register of systematic reviews (CRD42012002074). ${ }^{17}$

\section{Eligibility criteria}

As RCTs were known to be rare, primary studies of any design were sought that were conducted in primary care (including general practices and community pharmacies), recruited individuals aged $\geqslant 35$ years with no prior diagnosis of COPD (or provided sufficient data to separate out subjects with previously known and newly diagnosed COPD) and aimed to detect undiagnosed COPD confirmed by spirometry. Eligible screening tests included questionnaires, clinical examination, handheld flow meters, peak flow meters, decision aids/risk prediction models and chest radiography, either alone or in combination.

Note that there is no clear definition of the difference between case finding and screening. Some authors define screening as identifying asymptomatic patients and case finding as identifying patients with clinical disease, although the definition provided by the UK National Screening Committee includes structured case finding within screening. ${ }^{18}$ In this review, we sought studies that defined COPD using spirometric criteria,

\footnotetext{
${ }^{1}$ Public Health, Epidemiology \& Biostatistics, School of Health and Population Sciences, University of Birmingham, Edgbaston, Birmingham, UK.

Correspondence: RE Jordan (r.e.jordan@bham.ac.uk)
}

Received 14 February 2015; revised 14 June 2015; accepted 12 July 2015 


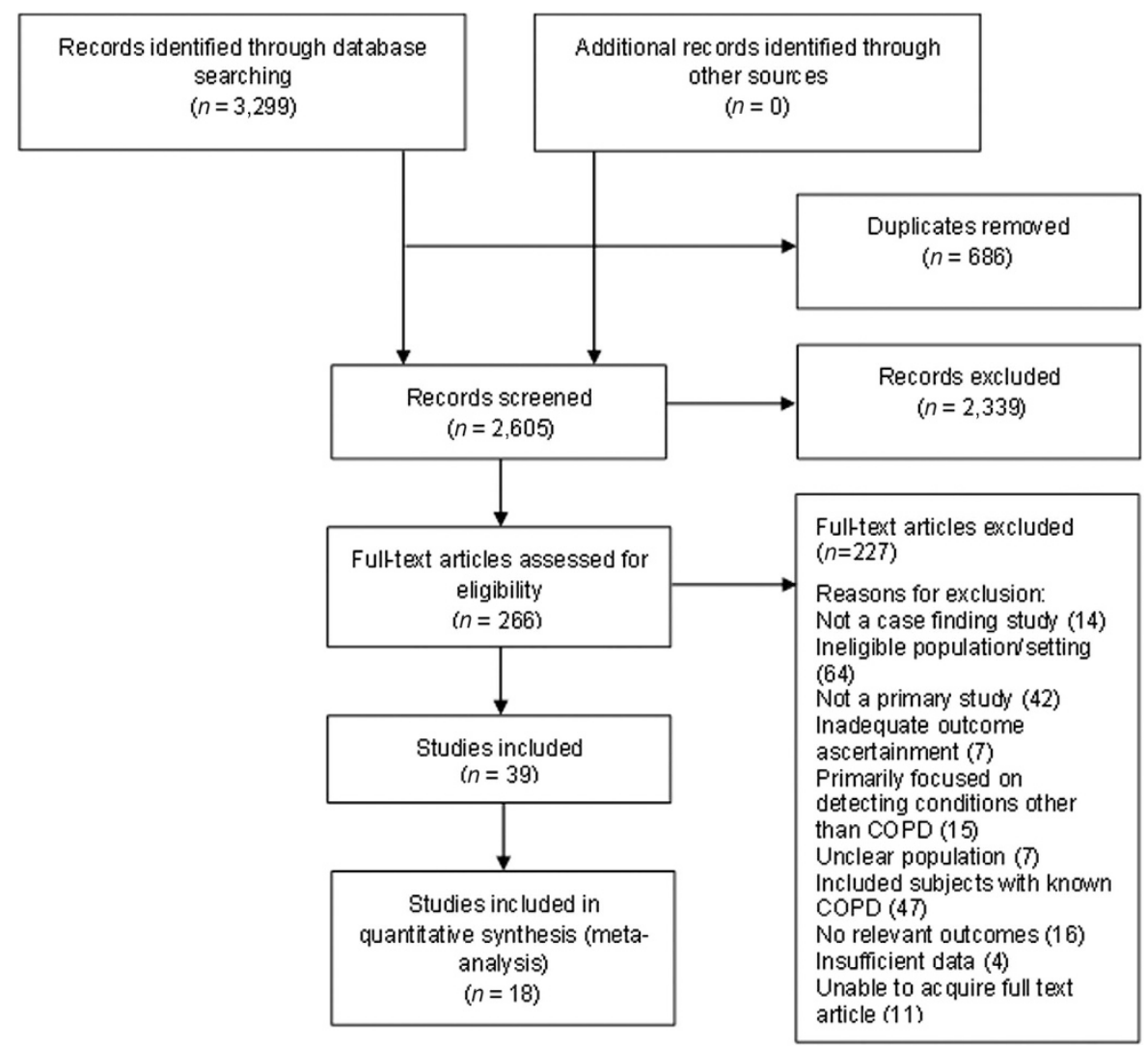

Figure 1. Article selection.

and draw particular attention to the studies in which the definition of COPD additionally incorporated clinical symptoms.

\section{Search strategy}

We searched Medline, Embase, CINAHL, Cochrane Central Register of Controlled Trials (CENTRAL), Database of Abstracts of Reviews of Effects, HTA Database and NHS Economic Evaluations Database from 1 March 2012 for the previous 15 years and performed an updated search in Medline and Embase up to December 2013. To identify grey literature, searches were also performed on Google Scholar, Turning Research into Practice, HTAi Vortal and DogPile, limited to the first 100 articles per search. No language restrictions were applied (Supplementary Table S1).

\section{Study selection and data extraction}

Titles and abstracts were screened independently by two reviewers. Full-text papers were obtained for all potentially relevant studies, and the eligibility criteria were applied independently, with disagreements resolved through discussion. Data were extracted on the characteristics of the selected population, approaches to recruitment, method of screening, number of participants newly diagnosed with COPD and numbers who were eligible, participated in screening and underwent diagnostic spirometry. In cases in which these data were not provided, we used information provided in the paper to derive them.

\section{Methodological quality assessment}

Included studies were assessed independently by two reviewers against criteria adapted from the QUADAS- $1^{19}$ and QUADAS- $2^{20}$ checklists, and the Cochrane risk of bias assessment tool for RCTs. ${ }^{21}$ Disagreements were resolved through discussion.

\section{Statistical analysis}

Results from comparative studies were described but not suitable for synthesis. We therefore used data from individual study arms of the trials together with the non-comparative studies to summarise uptake of tests and yields. Where there was sufficient methodological homogeneity, these were combined using random-effects meta-analyses. Forest plots were constructed to explore between-study heterogeneity in the yield, including differences in population characteristics, screening tests, diagnostic criteria and study design. All analyses were performed using Stata version 13.1 (Stata-Corp, College Station, TX, USA) and StatsDirect version 2.7.9 (Altrincham, Cheshire, UK).

\section{RESULTS}

Study selection

After removing duplicates, 2,605 citations were identified and 266 full-text articles were assessed for eligibility (Figure 1). A total of 39 studies were finally selected, from which 18 were included in meta-analyses. Studies that did not exclude patients with previously known COPD, or those that did not provide sufficient data to separate out new from existing diagnoses of COPD, were excluded.

\section{Overall study characteristics}

There were 2 individual RCTs, 1 cluster RCT, 1 non-randomised controlled trial, 25 single-arm before-after studies and 10 crosssectional test accuracy studies (Supplementary Table S2). Most of them were conducted in general practices $(n=34)$, two in community pharmacies and the remaining studies in either a health screening clinic $(n=1)$ or unclear primary care setting $(n=2)$. They evaluated the use of screening questionnaires $(n=13)$, handheld flow meters $(n=5)$ and direct invitation for diagnostic spirometry $(n=30)$. No studies evaluating other screening tests met the inclusion criteria.

\section{Comparative studies}

The comparative studies were highly heterogeneous, with major differences in trial design and comparators (Table 1). An RCT of nurse-led case finding among four general practices in Australia 


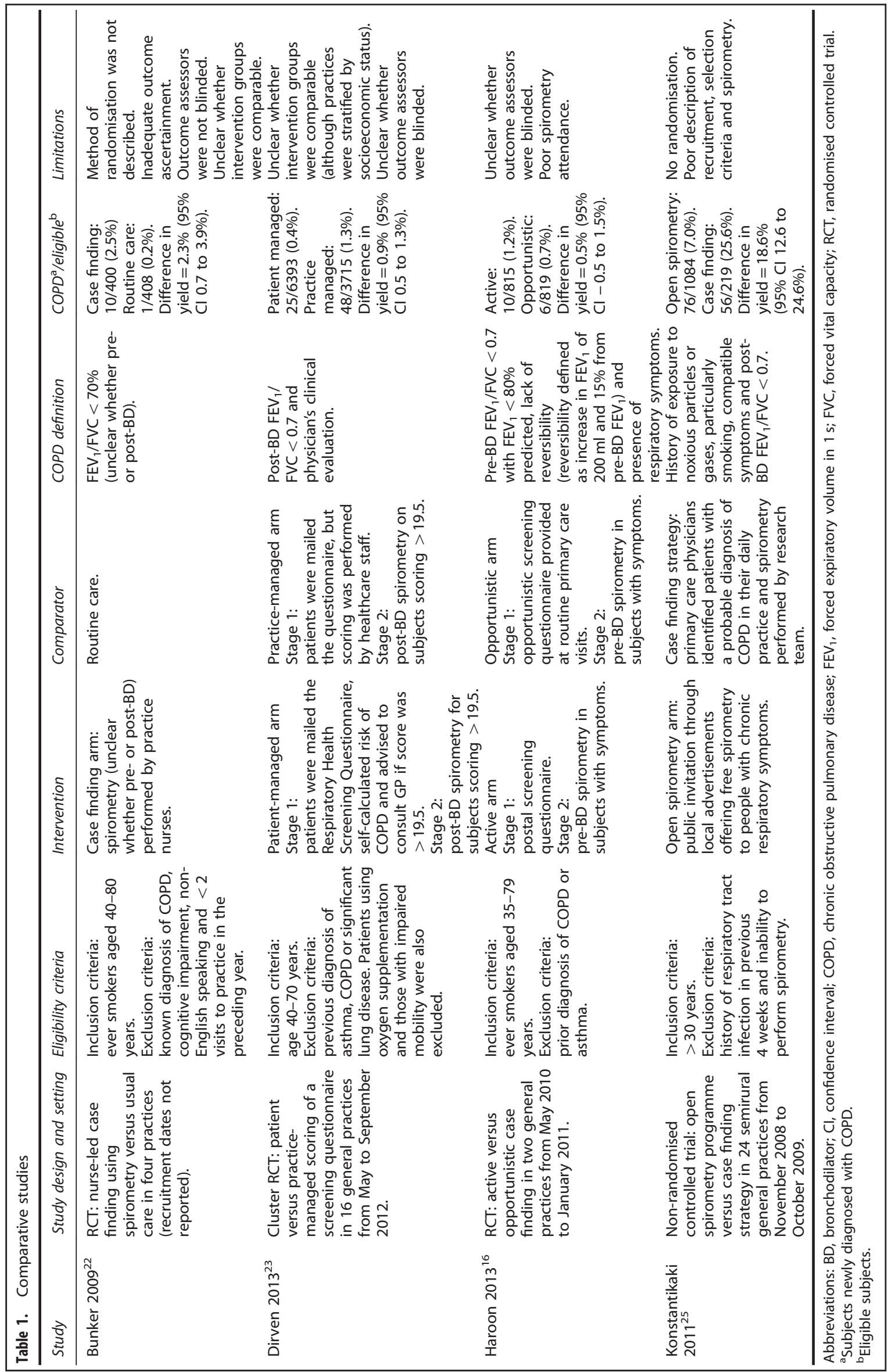




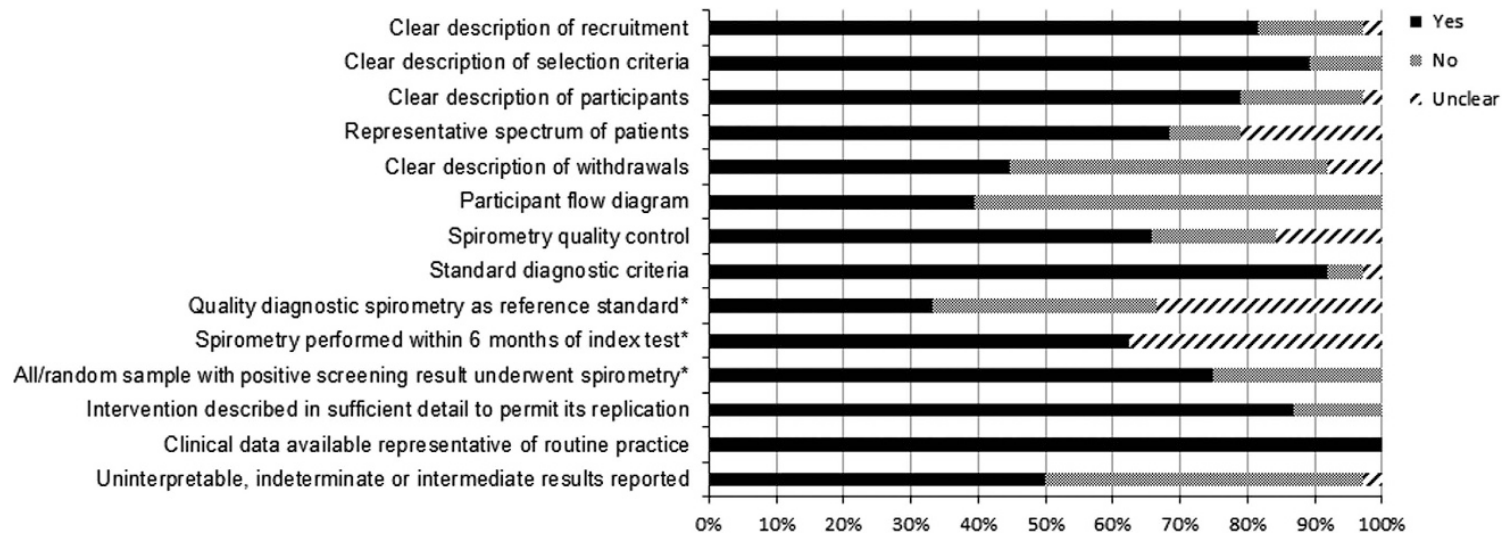

Figure 2. Quality assessment. '*' indicates only studies evaluating screening questionnaires and/or handheld flow meters.

showed that using written invitations to attend spirometry led to $2.3 \%$ (95\% confidence interval $(\mathrm{Cl}) 0.7$ to $3.9 \%$ ) higher yield of new cases than usual care. ${ }^{22}$ A cluster RCT in the Netherlands found that a practice-managed approach was more effective than patient self-scoring of a respiratory screening questionnaire before spirometry assessment (difference in yield $0.9 \%(0.5$ to $1.3 \%))^{23}$ Our own pilot trial comparing postal versus opportunistic screening using a respiratory questionnaire before diagnostic spirometry indicated that the former might lead to higher yield. ${ }^{24}$ However, the study lacked sufficient power to detect significant differences. Finally, a non-randomised trial found that offering spirometry to patients attending primary care suspected by their GP to have COPD resulted in a significantly higher yield than mass invitation to people with chronic respiratory symptoms (difference $18.6 \%(95 \% \mathrm{Cl} 12.6$ to $24.6 \%))^{25}$ These comparative studies suffered from problems of randomisation, ${ }^{22,25}$ inadequate blinding of assessors to intervention arms $\mathrm{s}^{22-25}$ and insufficient clarity about whether the populations in trial arms were comparable 22,23 (Supplementary Table S3). There was too much heterogeneity in their design and outcomes to combine their results.

Estimating the effect of different case finding approaches using all data from uncontrolled studies and single arms of trials

Methodological quality. The majority of included studies were uncontrolled single-arm studies and were highly heterogeneous. They generally provided a clear description of recruitment, selection criteria, characteristics of screened and clinically assessed participants and how spirometry was performed. Most of the studies used standard diagnostic criteria for COPD or airflow obstruction as the outcome. However, they often failed to report the complete eligible population, withdrawals, uninterpretable and indeterminate test results, participant flow or spirometry quality control procedures (Figure 2, Supplementary Tables S4a,b).

Recruitment and population selection. Participants were actively recruited through postal invitations, telephone calls and advertisements ( $n=17$ studies), were opportunistically invited at primary care consultations $(n=17)$ or were recruited through a combination of both approaches ( $n=2$; Table 2, Supplementary Table S2). Age (usually $\geqslant 40$ years) and a positive smoking history were the main eligibility criteria, although more than half of the studies included never smokers. A small number of studies specified additional eligibility criteria including the presence of respiratory symptoms ${ }^{26-29}$ or recent acute respiratory infections. ${ }^{30}$

Screening tests and diagnosis of COPD. Strategies for targeting those at high risk included using basic patient characteristics only (e.g., age and smoking status $(n=30))$, use of respiratory screening questionnaires $(n=13)$ and administration of handheld flow meters $(n=5)$ before diagnostic spirometry. Only nine studies included a clinical component to the case definition of COPD. All other studies used a purely physiological definition of COPD based on airflow limitation, most commonly a forced expiratory volume in $1 \mathrm{~s}$ to forced vital capacity ratio of $<70 \%$ (FEV $1 / F V C<0.7$; $n=30$ ). In addition, 13 used pre-bronchodilator spirometry for diagnosis, although the yields did not seem to significantly differ according to the use of pre- or post-bronchodilator testing.

Yield of new COPD cases with different approaches. Table 2 summarises the characteristics and yield (proportion of new cases) of all of the included studies for each case finding approach. With direct invitation for diagnostic spirometry, the overall proportion of all eligible subjects newly diagnosed with COPD (as defined by each study) ranged from 1.7 to $30.5 \%$ (18 studies; Table 2, Supplementary Tables S5 and S6). Most of them had mild-tomoderate disease, although in some studies a proportion (up to $37.2 \%$ ) of subjects had severe disease, which may have been related to the inclusion of a higher proportion of current smokers and patients with respiratory symptoms. Studies with highest yields were mainly test accuracy studies (which generally had higher methodological quality and possibly had more robust methods for inviting, assessing and following up participants) and those including only symptomatic patients.

Among 13 studies that tested screening questionnaires before diagnostic spirometry (Table 2, Supplementary Table S7), the COPD Diagnostic Questionnaire $^{14}$ (also referred to as the International Primary Airways Group Questionnaire and the Respiratory Health Screening Questionnaire) was most widely evaluated ( $n=5$ studies). Overall, new cases of COPD ranged from 0.4 to $22.3 \%$ of those eligible, again with the highest yields found in test accuracy studies.

Five (mostly test accuracy) studies evaluated screening with handheld flow meters before diagnostic spirometry (Table 2, Supplementary Tables S8 and S9). Overall, the yield ranged from 6 to $20 \%$ of those eligible, and all but one recruited patients opportunistically.

Because of the methodological heterogeneity, it was not possible to combine studies or draw firm conclusions, although incorporating an initial screening test before diagnostic assessment resulted in generally higher yield (percentage diagnosed with COPD out of all those referred for spirometry was 19-94\% for handheld flow meters, $14.3-42.1 \%$ with screening questionnaires and $4.1-40.2 \%$ with no pre-screening).

Exploring the effect of different target populations and recruitment strategies

We combined the results of studies including ever smokers, and a further subset among those already reporting respiratory symptoms because they appeared to be relatively homogeneous. 
Table 2. Studies evaluating spirometry, screening questionnaires and handheld flow meters

Characteristic

Range (no. of studies)

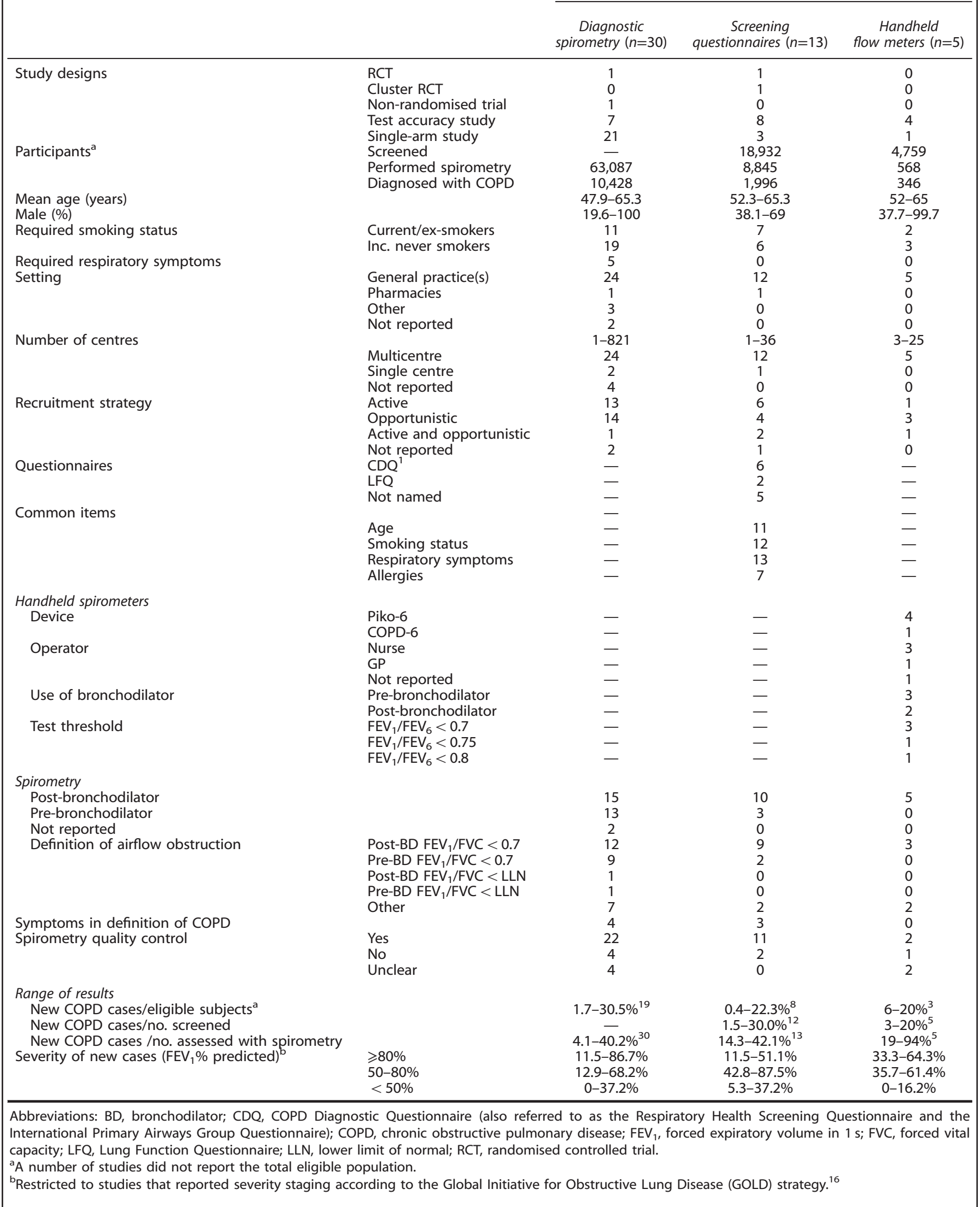




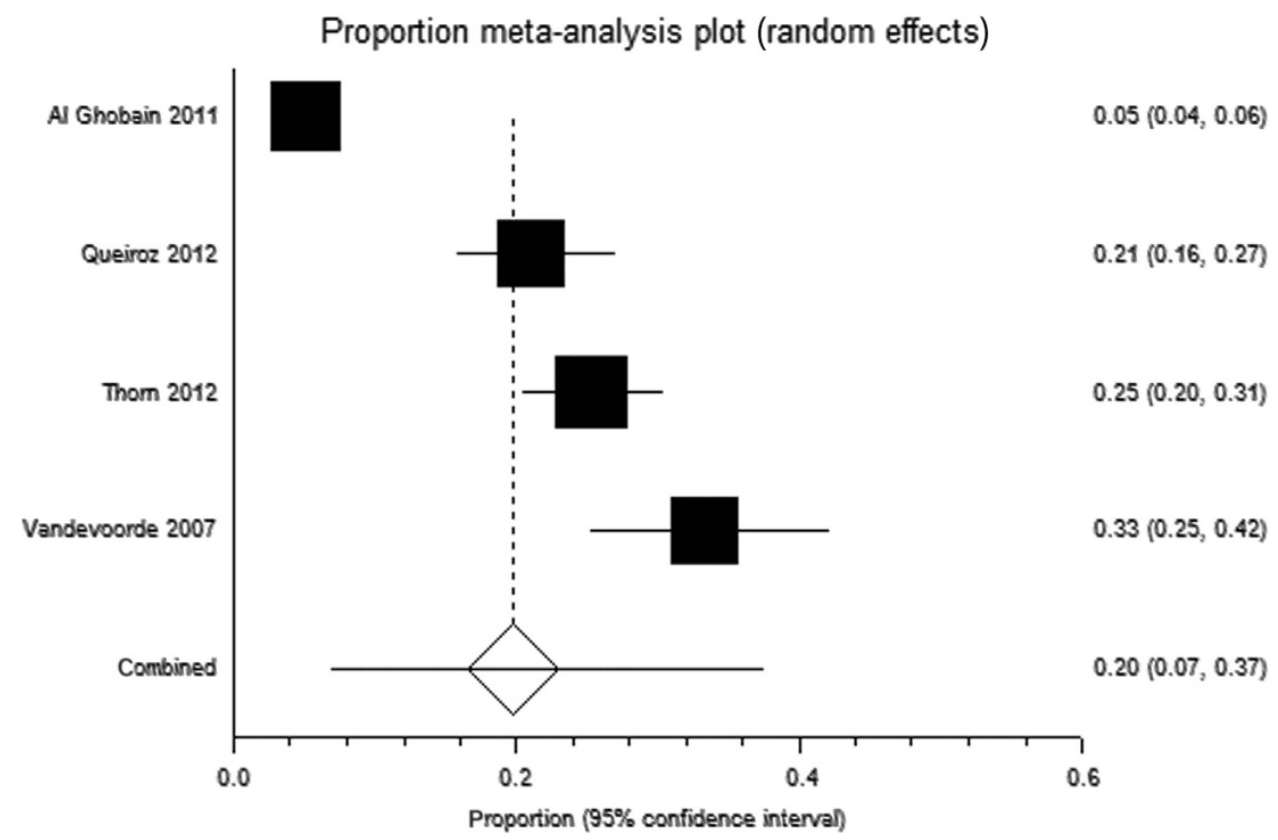

Figure 3. Random-effects meta-analysis of the proportion of eligible ever smokers diagnosed with COPD when opportunistically invited for spirometry (restricted to studies that defined airflow obstruction as $\mathrm{FEV}_{1} / \mathrm{FVC}<0.7$ ).

Uptake of screening/diagnostic tests

Opportunistic invitation of ever smokers at routine primary care attendances was associated with a significantly higher uptake of spirometry ( $97 \%(95 \% \mathrm{Cl} 90$ to $100 \%) ; n=5$ studies $\left.^{28,31-34}\right)$ than actively inviting ever-smoking patients by post (47\% (16 to $80 \%)$; $n=6$ studies $^{22,30,35-38}$ ), although Cls were wide (which may reflect heterogeneity in the populations being studied). Meta-analysis $\left(n=4\right.$ studies $^{23,24,35,39}$ ) showed an average response to postal questionnaires of $30 \%$ ( $95 \% \mathrm{Cl} 20$ to $41 \%$ ). There were insufficient data to estimate the response when questionnaires were distributed opportunistically at primary care attendances and to estimate the overall yield from either approach.

\section{Target populations}

From four similar studies, in which ever smokers aged $\geqslant 40$ years were opportunistically invited directly for diagnostic spirometry, $20 \%\left(95 \% \mathrm{Cl} 7\right.$ to $37 \%{ }^{31,33,40,41}$ ) were diagnosed with airflow obstruction (Figure 3), although Cls were wide and not all would necessarily have clinical disease. Restricting recruitment to ever smokers attending primary care and reporting a history of respiratory symptoms/infections provided a higher yield of $32 \%$ $\left(95 \% \mathrm{Cl} 21\right.$ to $44 \% ; n=3$ studies $\left.^{27,28,42}\right)$, although again these Cls were wide.

\section{DISCUSSION}

\section{Main findings}

This review incorporated evidence from 39 primary studies. The few comparative studies had a number of methodological limitations and were highly heterogeneous. They suggested that active case finding was likely to identify a greater number of patients with undiagnosed COPD compared with usual care, ${ }^{22}$ that scoring of screening questionnaires before diagnostic assessment should be practice-led rather than patient-led ${ }^{23}$ and that assessment of patients clinically suspected to have COPD is likely to result in higher yield than widespread invitation of the general public for screening. ${ }^{25}$ The remaining studies were noncomparative, with significant limitations in study design and the reporting of eligible populations, withdrawals, indeterminate results and spirometry quality control procedures. The lack of direct comparisons and the heterogeneity in populations, study design, definition of COPD and methods for estimating the yield limited our ability to draw firm conclusions about the most effective case finding approach.

However, indirect comparisons suggested that uptake for spirometry was higher when patients were invited opportunistically at routine primary care visits than with active postal invitation, and response to mailed screening questionnaires is likely to be $\sim 30 \%$. Studies that opportunistically screened ever smokers with a history of respiratory symptoms seemed to have a higher yield (although not statistically significant) than opportunistic studies among ever smokers in which symptoms were not considered. Yields from different approaches were highly variable, although pre-screening using either a questionnaire or handheld flow meter seemed to achieve a higher yield than direct invitation for diagnostic assessment. However, no studies had directly compared questionnaires with the use of handheld devices, nor considered respective costs. Finally, case finding is likely to uncover a significant burden of disease, and although the majority of this will be of mild-to-moderate severity a notable proportion ( $>10 \%$ in several studies) will have severe airflow obstruction.

Interpretation of findings in relation to previously published work The UK National Screening Committee recently recommended against screening for COPD because of a lack of relevant RCTs, and insufficient evidence on the optimal approach and the benefits of early treatment. ${ }^{12}$ They also concluded that opportunistic case finding among symptomatic individuals with more developed COPD was likely to be cost-effective and that it should continue. An older systematic review concluded that mass invitation for diagnostic spirometry (without prior pre-screening) could not be recommended, as large numbers of assessments would be needed to prevent a single COPD exacerbation, and would largely unveil patients with asymptomatic airflow obstruction for whom there are limited therapeutic options. ${ }^{13}$ Our findings concur with these points but also highlight several important methodological limitations that should be addressed in future trials evaluating case finding strategies. Furthermore, we found that although the majority of new patients had mild disease (which is arguably when 
secondary prevention may be most effective) a substantial proportion of those diagnosed are likely to have moderate-tosevere disease, with potential to benefit from evidence-based therapies. $^{43}$

Jithoo et al. $^{44}$ recently compared alternative case finding strategies, concluding that peak flow meters were a costeffective screening test for COPD. However, the peak expiratory flow rate was derived from quality-controlled spirometry measurements and may not necessarily reflect the accuracy of peak flow meters used in everyday clinical practice. Our search strategy did not identify studies directly evaluating peak flow meters for case finding. RCTs comparing the effectiveness of screening with peak flow meters against other screening tests such as questionnaires and handheld flow meters should therefore be considered.

Strengths and limitations of this study

We performed an extensive review of the literature and incorporated detailed evidence on 39 primary studies. However, there were very few RCTs, and heterogeneity among those identified precluded combining them. We therefore mainly relied on indirect comparisons, where potential bias arising from differences in study design and population characteristics made their combination inappropriate. Missing information on the size of the eligible population limited estimates of the uptake of screening and could potentially bias estimates obtained from meta-analyses. Furthermore, many of the combined results had wide confidence intervals and should be interpreted with caution. Unfortunately, with so few RCTs and so many single-arm studies, it was not possible to undertake the usual statistical analyses to test for publication bias. However, the search strategy was very comprehensive, and thus relevant RCTs are unlikely to have been missed.

Another important limitation was that most studies used a physiological definition of COPD based on spirometry, without clinical confirmation (ascertaining the presence of a compatible clinical history, including the presence of relevant symptoms). This is likely to overestimate the effectiveness (yield) of case finding, as a previous analysis undertaken by our group ${ }^{45}$ found that only about half of the individuals with undiagnosed airflow obstruction reported symptoms compatible with a clinical diagnosis of COPD. Identification of individuals with asymptomatic airflow obstruction is problematic in the absence of evidence-based recommendations to guide their management. ${ }^{13}$ In addition, the use of the fixed ratio for $\mathrm{FEV}_{1} / \mathrm{FVC}$ is controversial and many people believe this may also lead to overdiagnosis. ${ }^{46}$ Conversely, patients with undiagnosed COPD may underreport symptoms, as they often adapt to them over time until the quality-of-life is markedly affected, which may contribute to diagnostic delay. ${ }^{47}$

\section{Implications for future research, policy and practice}

Case finding through any approach is likely to uncover a substantial number of patients with undiagnosed COPD. With the current evidence, it is unclear whether opportunistic or active case finding results in a higher overall yield, although targeting patients with a history of respiratory symptoms or infections might improve the case finding yield. Pre-screening using either a questionnaire such as the COPD Diagnostic Questionnaire or handheld flow meter may reduce the number of diagnostic assessments needed compared with directly inviting all high-risk patients for diagnostic spirometry. However, it is unclear whether pre-screening with a handheld device would reduce the overall number of clinical contacts or improve the use of healthcare resources. Although the majority of patients identified through case finding will have mild-to-moderate disease, around one in five is likely to have severe disease with potential to benefit from being identified.
At the moment, opportunistic case finding is recommended as a cost-effective strategy by the National Institute for Health and Care Excellence (NICE). ${ }^{48}$ Our review concludes that focusing case finding opportunistically on those at high risk (based on age and previous smoking history), and reporting relevant clinical symptoms, is likely to improve the efficiency of case finding. However, there is insufficient evidence from well-conducted comparative studies to suggest which wider screening approach would result in higher yield, and there is insufficient evidence to support a more active approach to case finding.

No further information is needed from single-arm noncontrolled studies. Future studies should directly compare approaches, particularly addressing how yield could be further increased by targeting those at highest risk (e.g., by using risk prediction models), considering alternative recruitment strategies (opportunistic versus a more active approach for inviting those at high risk) and alternative screening tests (e.g., questionnaires, handheld flow meters, peak flow meters and risk prediction models, alone and in combination).

A definition of COPD should be used that is standardised and aligns with current recommendations, requiring clinical confirmation in addition to airflow limitation. ${ }^{11}$ They should also report withdrawals and indeterminate results and present this in a participant flow chart, including a description of the size and characteristics of the eligible population and those who received a screening and diagnostic assessment. They should also use strict quality control procedures for spirometry to avoid misdiagnosis. The findings of these studies will be important to enable economic evaluations of alternative case finding strategies to aid policymakers to make appropriate decisions on the most cost-effective approach.

\section{Conclusions}

There is extensive heterogeneity and few RCTs among studies evaluating case finding strategies for COPD in primary care, and it remains unclear as to which approach has the higher overall yield. Observational studies suggest that targeting specific sub-groups such as ever smokers with a history of respiratory symptoms may be more efficient, and using screening tests such as questionnaires may reduce the number of diagnostic assessments needed to identify a patient with COPD. High-quality RCTs are needed to make direct comparisons of alternative case finding strategies, including the target population, recruitment strategy and screening tests, taking into consideration the methodological limitations of previous studies highlighted in this review. There is currently inadequate evidence of effectiveness or cost-effectiveness for recommending any particular approach to case finding in primary care. There is also a need to evaluate the impact of case finding on clinical care and patient outcomes before firm recommendations can be made.

\section{ACKNOWLEDGEMENTS}

We thank Malcolm Price and Richard Riley for their advice on the statistical analysis; Clare Davenport and David Moore for their advice on the review protocol; Anne Fry-Smith for advice on the search strategy; Susan Bayliss, Xiaoying Wu, Kinga Malottki and Cristina Penaloza for translating foreign language articles; and Simon Stevens for acquiring the review articles.

\section{CONTRIBUTIONS}

The need for a review was conceived by PA and REJ. SH designed the protocol, performed the literature search, selected articles, extracted data, assessed the quality of articles, synthesised the results and wrote the manuscript. REJ advised on the protocol, selected articles, verified the extracted data, assessed the quality of articles, advised on the data synthesis and revised the manuscript. JOB-E selected articles, verified the extracted data and assessed the quality of articles. PA advised on the 
protocol, verified the extracted data, assessed the quality of articles, advised on the data synthesis and revised the manuscript. PA is the guarantor.

\section{COMPETING INTERESTS}

REJ and PA are principal investigators on the TargetCOPD trial, which evaluates the effectiveness of different approaches to case finding for COPD in primary care. TargetCOPD is funded by the National Institute for Health Research under its Programme Grants for Applied Research Programme (Grant Reference Number RP-PG-0109-10061).

\section{FUNDING}

This paper presents independent research funded by the National Institute for Health Research (NIHR). The views expressed are those of the authors and not necessarily those of the NHS, the NIHR or the Department of Health. SH is funded by an NIHR doctoral fellowship (DRF-2011-04-064). REJ was funded by an NIHR post-doctoral fellowship (pdf/01/2008/023). The funders had no role in study design, data collection and analysis, decision to publish or preparation of the manuscript.

\section{REFERENCES}

1 Lozano R, Naghavi M, Foreman K, Lim S, Shibuya K, Aboyans V et al. Global and regional mortality from 235 causes of death for 20 age groups in 1990 and 2010: a systematic analysis for the Global Burden of Disease Study 2010. Lancet 2012; 380: 2095-12.

2 Murray CJ, Vos T, Lozano R, Naghavi M, Flaxman AD, Michaud C et al. Disability-adjusted life years (DALYs) for 291 diseases and injuries in 21 regions, 1990-2010: a systematic analysis for the Global Burden of Disease Study 2010 Lancet 2012; 380: 2197-2223.

3 Britton $M$. The burden of COPD in the UK: results from the Confronting COPD survey. Respir Med 2003; 97 (Suppl C): S71-S79.

4 Soriano J, Zielinski J, Price D. Screening for and early detection of chronic obstructive pulmonary disease. Lancet 2009; 374: 721-732.

5 Fromer L. Diagnosing and treating COPD: understanding the challenges and finding solutions. Int J Gen Med 2011; 4: 729-739.

6 Kesten S, Ulrich J, Rich D. Public awareness of chronic obstructive pulmonary disease. Chest 2003; 124(4 MeetingAbstracts): 167S.

7 Soriano JB, Calle M, Montemayor T, Alvarez-Sala JL, Ruiz-Manzano J, Miravitlles M. The general public's knowledge of chronic obstructive pulmonary disease and its determinants: current situation and recent changes. Arch Bronconeumol 2012; 48 308-315.

8 Jones RCM, Price D, Ryan D, Sims EJ, von Ziegenweidt J, Mascarenhas L et al. Opportunities to diagnose chronic obstructive pulmonary disease in routine care in the UK: a retrospective study of a clinical cohort. Lancet Respir Med 2014; 2: 267-276.

9 Calderon-Larranaga A, Carney L, Soljak M, Bottle A, Partridge M, Bell D et al. Association of population and primary healthcare factors with hospital admission rates for chronic obstructive pulmonary disease in England: national crosssectional study. Thorax 2011; 66: 191-196.

10 Bastin AJ, Starling L, Ahmed R, Dinham A, Hill N, Stern M et al. High prevalence of undiagnosed and severe chronic obstructive pulmonary disease at first hospital admission with acute exacerbation. Chron Respir Dis 2010; 7: 91-97.

11 Global Initiative for Chronic Obstructive Lung Disease. Global strategy for the diagnosis, managment, and prevention of chronic obstructive pulmonary disease Global Initiative for Chronic Obstructive Lung Disease 2015. Available from: http:// www.goldcopd.org/.

12 Cartwright S. An evaluation of screening for COPD against the National Screening Committee criteria 2012.

13 Lin K, Watkins B, Johnson T, Rodriguez JA, Barton MB. US Preventive Services Task Force Screening for chronic obstructive pulmonary disease using spirometry: summary of the evidence for the US. Preventive Services Task Force. Ann Intern Med 2008; 148: 535-543.

14 Price DB, Tinkelman DG, Nordyke RJ, Isonaka S, Halbert RJ. COPD Questionnaire Study Group Scoring system and clinical application of COPD diagnostic questionnaires. Chest 2006; 129: 1531-1539.

15 Frith P, Crockett A, Beilby J, Marshall D, Attewell R, Ratnanesan A et al. Simplified COPD screening: validation of the PiKo-6(R) in primary care. Prim Care Respir $J$ 2011; 20: 190-198.

16 Haroon SM, Adab PA, Jordan RE. Case finding for COPD in primary care: a systematic review. Prim Care Respir J 2012; 21: 354-357.

17 PROSPERO: International prospective register of systematic reviews York: Centre for Reviews and Dissemination, University of York; 2014. Available from: http:// www.crd.york.ac.uk/PROSPERO/.
18 UK Screening Portal London: Public Health England; 2014 [cited 2014 06.12.2014] Available from: http://www.screening.nhs.uk/screening.

19 Whiting P, Rutjes AW, Dinnes J, Reitsma J, Bossuyt PM, Kleijnen J. Development and validation of methods for assessing the quality of diagnostic accuracy studies. Health Technol Assess 2004; 8, iii, 1-234.

20 Whiting PF, Rutjes AW, Westwood ME, Mallett S, Deeks JJ, Reitsma JB et al. QUADAS-2: a revised tool for the quality assessment of diagnostic accuracy studies. Ann Intern Med 2011; 155: 529-536.

21 Higgins J, Green SP. Cochrane Handbook for Systematic Reviews of Interventions. Wiley-Blackwell: Oxford, UK, 2008.

22 Bunker J, Hermiz O, Zwar N, Dennis SM. Feasibility and efficacy of COPD case finding by practice nurses. Aust Fam Physician 2009; 38: 826-830.

23 Dirven J, Tange H, Muris J, van Haaren K, Vink G, van Schayck O. Early detection of COPD in general practice: patient or practice managed? A randomised controlled trial of two strategies in different socioeconomic environments. Prim Care Respir J 2013; 22: 331-337.

24 Haroon S, Adab P, Griffin C, Jordan R. Case finding for chronic obstructive pulmonary disease in primary care: a pilot randomised controlled trial. $\mathrm{Br} J$ Gen Pract 2013; 63: e55-e62.

25 Konstantikaki V, Kostikas K, Minas M, Batavanis G, Daniil Z, Gourgoulianis KI et al. Comparison of a network of primary care physicians and an open spirometry programme for COPD diagnosis. Respir Med 2011; 105: 274-281.

26 Broekhuizen BD, Sachs AP, Hoes AW, Moons KG, van den Berg JW, Dalinghaus WH et al. Undetected chronic obstructive pulmonary disease and asthma in people over 50 years with persistent cough. Br J Gen Pract 2010; 60: 489-494.

27 Kogler H, Metzdorf N, Glaab T, Welte T. Preselection of patients at risk for COPD by two simple screening questions. Respir Med 2010; 104: 1012-1019.

28 Lokke A, Ulrik CS, Dahl R, Plauborg L, Dollerup J, Kristiansen LC et al. Detection of previously undiagnosed cases of COPD in a high-risk population identified in general practice. COPD 2012; 9: 458-465.

29 Ulrik CS, Lokke A, Dahl R, Dollerup J, Hansen G, Cording PH et al. Early detection of COPD in general practice. Int J Chron Obstruct Pulmon Dis 2011; 6 123-127.

30 Sandelowsky H, Stallberg B, Nager A, Hasselstrom J. The prevalence of undiagnosed chronic obstructive pulmonary disease in a primary care population with respiratory tract infections-a case finding study. BMC Fam Pract 2011; 12: 122-131.

31 Al Ghobain M, Al-Hajjaj MS, Wali SO. Prevalence of chronic obstructive pulmonary disease among smokers attending primary healthcare clinics in Saudi Arabia. Ann Saudi Med 2011; 31: 129-133.

32 Leuppi JD, Miedinger D, Chhajed PN, Buess C, Schafroth S, Bucher HC et al. Quality of spirometry in primary care for case finding of airway obstruction in smokers. Respiration 2010; 79: 469-474.

33 Vandevoorde J, Verbanck S, Gijssels L, Schuermans D, Devroey D, De Backer J et al. Early detection of COPD: A case finding study in general practice. Respir Med 2007 101: 525-530.

34 van Schayck C, Loozen J, Wagena E, Akkermans R, Wesseling G. Detecting patients at a high risk of developing chronic obstructive pulmonary disease in general practice: cross sectional case finding study. BMJ 2002; 324: 1370.

35 Freeman D, Nordyke RJ, Isonaka S, Nonikov DV, Maroni JM, Price D et al. Questions for COPD diagnostic screening in a primary care setting. Respir Med 2005; 99: 1311-1318.

36 Geijer RMM, Sachs APE, Hoes AW, Salome PL, Lammers JWJ, Verheij TJM. Prevalence of undetected persistent airflow obstruction in male smokers 40-65 years old. Fam Pract 2005; 22: 485-489.

37 Kotz D, Nelemans P, van Schayck CP, Wesseling GJ. External validation of a COPD diagnostic questionnaire. Eur Respir J 2008; 31: 298-303.

38 Stratelis G, Jakobsson P, Molstad S, Zetterstrom O. Early detection of COPD in primary care: screening by invitation of smokers aged 40 to 55 years. $\mathrm{Br} \mathrm{J}$ Gen Pract 2004; 54: 201-206.

39 Dirven JAM, Tange HJ, Muris JWM, van Haaren KMA, Vink G, van Schayck OCP. Early detection of COPD in general practice: implementation, workload and socioeconomic status. A mixed methods observational study. Prim Care Respir J 2013; 22: 338-343.

40 de Queiroz MCDAM, Moreira MAC, Rabahi MF. Underdiagnosis of COPD at primary health care clinics in the city of Aparecida de Goiania, Brazil. J Bras Pneumol 2012; 38: 692-699.

41 Thorn J, Tilling B, Lisspers K, Jorgensen L, Stenling A, Stratelis G. Improved prediction of COPD in at-risk patients using lung function pre-screening in primary care: a real-life study and cost-effectiveness analysis. Prim Care Respir J 2012; 21: 159-166.

42 Ulrik CS, Lokke A, Dahl R, Dollerup J, Hansen G, Cording PH et al. Early detection of COPD in general practice. Int J Chron Obstruct Pulmon Dis 2011; 6: 123-127.

43 Jordan R, Lam K-B, Cheng K, Miller M, Marsh J, Ayres J et al. Case finding for chronic obstructive pulmonary disease: a model for optimizing a targeted approach. Thorax 2010; 65: 492-498. 
44 Jithoo A, Enright PL, Burney P, Buist AS, Bateman ED, Tan WC et al. Case-finding options for COPD: results from the Burden of Obstructive Lung Disease study. Eur Respir J 2013; 41: 548-555.

45 Craig R, Hirani V, (eds). The Health Survey for England 2009. The Information Centre: London, UK, 2010.

46 Hardie JA, Buist AS, Vollmer WM, Ellingsen I, Bakke PS, Morkve O. Risk of overdiagnosis of COPD in asymptomatic elderly never-smokers. Eur Respir J 2002; 20: 1117-1122.

47 van den Boom G, Rutten-van Molken MP, Tirimanna PR, van Schayck CP, Folgering $\mathrm{H}$, van Weel $\mathrm{C}$. Association between health-related quality of life and consultation for respiratory symptoms: results from the DIMCA programme. Eur Respir J 1998; 11: 67-72.
48 Chronic Obstructive Pulmonary Disease: Management of Chronic Obstructive Pulmonary Disease in Adults in Primary and Secondary Care. National Institute for Health and Clinical Excellence: London, UK, 2010.

(i) This work is licensed under a Creative Commons Attribution 4.0 International License. The images or other third party material in this article are included in the article's Creative Commons license, unless indicated otherwise in the credit line; if the material is not included under the Creative Commons license, users will need to obtain permission from the license holder to reproduce the material. To view a copy of this license, visit http://creativecommons.org/licenses/ by/4.0/

Supplementary Information accompanies the paper on the npj Primary Care Respiratory Medicine website (http://www.nature.com/npjpcrm) 